Proceedings of Anticancer Research

Research Article

\title{
The Clinical Study of Multigene Combination Test to Guide Chemotherapy Combined with Targeted Therapy in Patients with Advanced Gastrointestinal Tumors
}

Zhisheng Zhang*, Gaoyang Chen, Qin Dai

Taizhou Second People's Hospital, Taizhou City 225500, Jiangsu Province, China

\begin{abstract}
Objective: To study the clinical effects of multigene combination test to guide chemotherapy combined with targeted therapy in patients with advanced gastrointestinal tumors. Methods: The samples were selected from 60 patients with advanced gastrointestinal tumors admitted to our hospital from March 2019 to July 2020, and were divided into a study group and a control group using a random number table model; patients in the control group did not undergo genetic testing and FOLLOX 4 +PD-1 chemotherapy, while patients in the study group underwent TYMS, ERCC1, EGFR, and KRAS and VEGF gene expression levels test, and the sensitive treatment plan was determined based on the test results, and the clinical indexes were compared between the two groups. Results: By comparing the total effective rate, survival time, and time to disease progression of chemotherapy in the two groups, the study group has a significant advantage $(\mathrm{P}<0.05)$. Conclusion: The combination of chemotherapy and targeted therapy for advanced gastrointestinal tumor patients can improve the efficiency of chemotherapy and prolong the time of disease progression and survival, which is worthy of comprehensive promotion.
\end{abstract}

Keywords: Multigene combination test; Advanced gastrointestinal tumors; Chemotherapy; Targeted therapy

Publication date: September, 2020

Publication online: 30 September, 2020

*Corresponding author: Zhisheng Zhang, zzs13952683766@163.com
Stomach cancer is one of the most frequent malignant tumors in the digestive system. According to relevant statistics, the incidence rate of stomach cancer in China is $31.38 / 100,000$, and the mortality rate is $22.13 / 100,000^{[1]}$. Stomach cancer patients have no typical clinical symptoms in the early stage, and most of them are in the advanced stage when they are diagnosed, so they miss the best time for radical surgery and need systemic chemotherapy. The total effective rate of chemotherapy for gastric cancer is less than $50 \%$, and the 5 -year survival rate of patients is less than $7 \%$, so the clinical efficacy needs to be improved ${ }^{[2]}$. How to adopt an effective plan to predict the effect of chemotherapy, reduce ineffective chemotherapy, and decrease the incidence of adverse reactions is the hot issue in clinical research at this stage.

Recent studies have shown that the effects of chemotherapy in gastric cancer are correlated with drug mechanisms and drug-sensitive genes. Excision repair cross complement 1 (ERCC1), the key gene in the nucleic acid resection repair system, can be used as the main biomarker to assess the sensitivity of oxaliplatin. There was a negative correlation between the high expression of thymidylate synthase (TYMS) gene with the patients' sensitivity to chemotherapy and prognosis. Cetuximab molecularly targeted therapy is ineffective in patients with KRAS gene mutations ${ }^{[3]}$. The multigene combination test can provide a reference for determining the sensitivity of chemotherapy and targeted therapy for patients with advanced gastrointestinal tumors. This study summarizes the basic data of relevant patients in our hospital and investigates and analyzes the issues related to the specific application of a multigene combination test. 


\section{Materials and methods}

\subsection{Materials}

The selected study samples were 60 patients admitted to our hospital during March 2019-July 2020, who were diagnosed with advanced gastrointestinal tumors by pathological diagnostic tests, and had lesion tissues that could be accurately measured by MRI and CT. Their expected survival time is more than 3 months, and they donot have any cardiopulmonary and liver/kidney function abnormalities. All of them were given informed consent to the study content. Patients were divided into a study group and a control group using a random number table model. According to the basic data of the patients from two groups, the ratio of males to females in the study group was 16:14, and the mean patient age was $62.28 \pm 1.06$ years(range $48-77$ years); the ratio of males to females in the control group was 17:13, and the mean patient age was $62.77 \pm 1.25$ years(range $50-78$ years). All the baseline data had no adverse effect on the results of this study $(\mathrm{P}>0.05)$.

\subsection{Methods}

The combination of FOLFOX4 with PD-1 was administered to patients in the control group without a multigene combination test. The FOLFOX4 regimen included oxaliplatin, aldehyde-folic acid, and fluorouracil. Oxaliplatin was administered on day 1 at the dose of $85 \mathrm{mg} / \mathrm{m} 2$ intravenously, aldehyde-folic acid $200 \mathrm{mg} / \mathrm{m} 2$ was given intravenously on treatment day 1 and 2, and fluorouracil at the dose of $1400 \mathrm{mg} / \mathrm{m} 2$ was administered on day 1 and 2. By using a portable infusion pump IV drip, fluorouracil at the dose of 1200 $\mathrm{mg} / \mathrm{m} 2$ was administered continuously for $22 \mathrm{~h}$. Since the first day, a mixture of $500 \mathrm{ml}$ saline and $200 \mathrm{mg}$ PD-1 was administered intravenously for $2 \mathrm{~h}$ every day.

The gene expression levels (TYMS, ERCC1, EGFR, KRAS, and VEGF) of patients in the study group were tested, and sensitive treatment plans were determined based on the test results. $10 \mathrm{ml}$ of the venous blood sample was collected from each patient, and the physician detected the mRNA expression levels of these gastrointestinal tumor genes according to the operation of the kit: Real-time fluorescence PCR kit, cDNA synthesis kit, total RNA extraction kit, pGM-T extraction kit. According to the specific value, the gene expression level is divided into no expression, low expression, high expression, etc. If the patient has low expression of VEGF, bevacizumab is treated; if the patient has wild-type KRAS, cetuximab is used; if the patient has high expression of EGFR, irinotecan is selected; if the patient has low expression of ERCC1, oxaliplatin is administered; if the patient has low expression of TYMS, 5-Fu is chosen.

\subsection{Evaluation criteria}

Two months after treatment, the total effective rate of chemotherapy, survival time, and time of disease progression of patients from the two groups were compared.

\subsection{Statistical methods}

SPSS 23.0 software was used to calculate data. All data were expressed as mean $\pm \mathrm{SD}(\bar{x} \pm \mathrm{s})$, the $\mathrm{t}$ test was used to compare means from two groups, and the test method is $\chi^{2}$. $\mathrm{P}<0.05$ was identified to be statistically significant.

\section{Results}

By comparing the overall effectiveness of chemotherapy, survival time, and time to disease progression between the two groups, the study group had a significant advantage $(\mathrm{P}<0.05)$.

\section{Discussion}

Gastrointestinal tumors, one kind of digestive system tumors, have a high clinical incidence. In 2015, the estimated number of gastrointestinal tumors in China was 3.763 million, the estimated number of deaths was 1.910 million, and about $20 \%$ of patients had

Table 1. Comparison of the overall effectiveness of chemotherapy, survival time, and time to disease progression between the two groups

\begin{tabular}{|c|c|c|c|}
\hline Group & $\begin{array}{l}\text { Overall effectiveness of } \\
\text { chemotherapy }\end{array}$ & survival time (month) & $\begin{array}{l}\text { time to disease progression } \\
\text { (month) }\end{array}$ \\
\hline Study group $(\mathrm{n}=30)$ & $18(60.0)$ & $15.59 \pm 2.04$ & $7.54 \pm 1.28$ \\
\hline Control group $(n=30)$ & $10(33.3)$ & $12.31 \pm 1.85$ & $6.11 \pm 1.07$ \\
\hline$t$ value & 4.285 & 6.523 & 4.694 \\
\hline$P$ value & 0.038 & 0.000 & 0.000 \\
\hline
\end{tabular}


developed distant metastasis at the time of diagnosis. The conventional clinical treatment for gastrointestinal tumors is targeted therapy and chemotherapy. At this stage, the gold standard of chemotherapy for advanced gastrointestinal tumors is still lacking. In order to improve its clinical efficacy and safety, physicians should ensure optimal efficacy, best fit the individual characteristics of patients, and minimize toxic and side effects when determining the specific chemotherapy $\operatorname{plan}^{[4]}$.

According to the data analysis in this study, the total efficiency, survival time, and time to disease progression of patients in the study group have obvious advantages compared to the control group, so multi-gene combination assay to guide chemotherapy combined with targeted therapy has outstanding clinical value. With the significant progress of pharmacogenomics and molecular biology of tumors, multiple causes and heterogeneity of gastrointestinal tumors are gradually better understood in medical research, so individualized precision therapy has been a new development direction of gastrointestinal tumor treatment ${ }^{[5]}$. Relevant studies have shown that the combination of trastuzumab with chemotherapy has a significant therapeutic effect in patients with HER-2-positive advanced gastrointestinal tumors. The proportion of HER-2 overexpression and gene amplification in patients with advanced gastrointestinal tumors is relatively low, ranging from $7 \%$ to $34 \%$, and there is a lack of individualized chemotherapy regimen for patients with HER-2 negative. Recent studies have shown that chemotherapy and targeted therapy based on ERCC1, TYMS, and TUBB3 expression levels can significantly improve the efficacy of chemotherapy and prolong the survival time in patients with advanced gastrointestinal tumors.

In this study, we focused on detecting the gene expression levels of TYMS, ERCC1, EGFR, KRAS, and VEGF, and determined the chemotherapy regimens based on the results. (1) ERCC1: an important member of human DNA shear repair, whose main role is to rapidly repair DNA damage. Its specific expression level can have a great impact on the DNA repair process. The efficacy of platinum-based chemotherapy is negatively correlated with the expression level of ERCC1, so detecting the expression level of ERCC1 before platinum-based chemotherapy can improve the precision of platinum-based drug selection, and thus improve the efficacy of chemotherapy and patients, survival time. (2) TYMS: a rate-limiting enzyme that catalyzes the synthesis of pyrimidine nucleotides and is the main target enzyme for fluorouracil and other anticancer drugs ${ }^{[6]}$. Relevant data show that the chemotherapeutic effect of fluorouracil drugs is correlated with the expression level of TYMS, for example, the low expression level of TYMS gene can trigger a decrease in resistance to $5-\mathrm{Fu}$ in the patients, thus improving the therapeutic effect of chemotherapy and prolonging the survival period. (3) EGFR: a transmembrane receptor with tyrosine kinase activity, is overexpressed in rectal cancer. Its expression level correlates with tumor aggressiveness and abnormal proliferation. EGFR can activate Ras-MAPK and PI3KAkt pathways, enabling them to complete biological signaling. (4) KRAS: a key link in the EGFR signaling pathway. Its main expression product is ras, which can act as an intermediary to transmit extracellular signals to the inside of cells via EGFR. Gene mutation in KRAS can lead to the continuous activation of ras protein, which can activate the EGFR downstream signaling pathway, thus completing anti-apoptotic and proliferative effects ${ }^{[7]}$. Recent studies have shown that EGFR and its downstream signaling pathway play an important biological role in the repair of DNA doublestranded damage in tumor cells and that DNA doublestranded damage is the main way to kill tumor radiation damage. The repair of damage by EGFR directly determines the difference in radiosensitivity of tumor cells, and the high expression of EGFR and KRAS gene mutation can induce radiation injury repair in tumors, leading to radiation resistance and affecting the effect of chemotherapy ${ }^{[8]}$.

So the combination of chemotherapy and targeted therapy for patients with advanced gastrointestinal tumors can improve the efficiency of chemotherapy, time to progression of serious diseases, and survival time, which is worthy of comprehensive promotion. At the same time, this study is not perfect, because it is under the influence of many factors, and the total number of patients consulted is small. Therefore, the specific application of the multigene combination test needs further analysis.

\section{References}

[1] Ma YJ, Qiu WS. Bioinformatics-based analysis of ENC1 expression and its clinical significance in gastrointestinal tumors[J]. Clinical Advances, 2020, 10(6): 1032-1038.

[2] Meng K, Yu XQ, Wu Q, et al. Meta-analysis of RAD51135 locus gene polymorphism and gastrointestinal tumor susceptibility[J]. Journal of Wuhan University (Medical Edition), 2020, 41(1): 58-64.

[3] Liu CF, Sun NN, Shi M, et al. Progress in the diagnosis of gastrointestinal tumors by miR-17-92 gene cluster in peripheral blood [J]. World Updated Medical Information Digest (Continuous Electronic Journal), 2019, 19(20): 82-83, 88.

[4] Wang RM, Wang KK, Qu XJ, et al. Efficacy and safety analysis 
of UGT1A1 gene polymorphism and irinotecan in the treatment of gastrointestinal tumors[J]. Electronic Journal of Comprehensive Cancer Therapy, 2018, 4(3): 51-56.

[5] Chen HY, Yang XB, Xu DK. Progress in the prediction of efficacy and prognostic value of new biomarkers in gastrointestinal tumors[J]. Diagnostic Theory and Practice, 2019, 18(6): 704710.

[6] Ping $\mathrm{Y}, \mathrm{Wu} \mathrm{XD}$, Chen B, et al. Effect of normal c-CBL transfection on imatinib-resistant gastrointestinal stromal tumor cells[J]. Chinese Journal of Tumor Control, 2020, 27(12): 939-
943.

[7] Ma XL, Yang YZ, Wang J, et al. Vitamin D receptor gene polymorphism and sensitivity of chemotherapy in gastrointestinal tumors[J]. Ningxia Medical Journal, 2018, 40(3): 231-233.

[8] Shi SS, Qin S, Ye SB, et al. Clinical and molecular pathological features of 26 cases of sporadic SDH-deficient gastrointestinal stromal tumors[J]. Journal of Clinical and Experimental Pathology, 2020, 36(6): 631-635. 